\title{
Viscous damping of nanoscale resonators for gas composition analysis
}

\author{
Yang $\mathrm{Xu}^{1}, \mathrm{~J}-\mathrm{T}$. Lin ${ }^{2}$, Bruce W. Alphenaar ${ }^{2}$ and Robert S. Keynton ${ }^{1,3}$ \\ ${ }^{1}$ Department of Mechanical Engineering, University of Louisville, Louisville, KY \\ ${ }^{2}$ Department of Electrical and Computer Engineering, University of Louisville, Louisville, KY \\ ${ }^{3}$ Department of Bioengineering, University of Louisville, Louisville, KY
}

\begin{abstract}
The damping effect of various gas environments on a nanoscale resonator is investigated for gas composition analysis. The resonance frequency shift, due to the viscous damping of the cantilever, is found to be directly related to the molar mass of the gas, thereby providing a method to determine the composition of the gas environment. The advantages of this gas analysis method are that it is simple, repeatable, reversible and not limited to reactive gases. The influences of the temperature and the vibration amplitude on the resonance frequency shift are also discussed. Finally, the use of a Raman based spectroscopy technique to determine the temperature of the resonator is described.
\end{abstract}

Keywords-Viscous damping; resonator; gas sensor; lateral vibration; Piezoresistive detection; Raman spectra

Micro/nano resonator gas sensors have generated a great deal of interest due to their ability to detect minute gas concentrations [1-3]. Typically, these sensors detect the shift in resonance frequency which is due to the chemical reaction between the sensed gas and the resonator, such as adsorption or desorption of gas particles or intercalation of gas into the cantilever lattice. The usefulness of microresonator gas sensors is then limited by the availability of suitable gas reaction mechanisms, and by the irreversibility of the detection process. On the other hand, the operation of reaction-based microresonator gas sensors is mostly restricted to near vacuum conditions because the non-chemical interaction of the microresonator with the gaseous environment, via the damping force of the gas particles on the cantilever, is neglected. While this limitation is generally recognized, overlooked is the fact that this same damping force can itself be used as a means of non-reactive gas sensing.

Here, we demonstrate the use of the damping force for gas sensing in a silicon lateral vibrating nanoscale resonator. The amplitude of the resonance signal and the resonance frequency are both shown to be systematically dependent on the molar mass of the gas environment at a fixed pressure, which provides a viable method for gas identification without involving reactions between the gas and resonator. The device developed in this study can be operated at atmospheric pressure. The sensor can also be integrated into a chip using MEMS techniques because the resonator is electrostatically activated and the vibration is detected using piezoresistive method [4-7]. The damping force detection method can be used for sensing inert gases, measuring the composition of binary gas mixtures or monitoring changes in the environment as a function of time. Although this technique is not suitable for detecting small quantities of gas, it is simple, repeatable and reliable compared to reaction-based detection methods and provides a means to sense non-reactive gases.

Fig. 1 shows the T-shape resonator used in our study. The base of the resonator is fixed at both ends by electrical contacts and acts as a piezoresistive sensing element. The cantilever beam extends from the base and is driven by a capacitivelycoupled gate electrode. The resonator is defined from the top silicon layer of a silicon-on-insulator (SOI) wafer using electron beam lithography and deep-reactive-ion etching (DRIE). For DRIE, a $\mathrm{C}_{4} \mathrm{H}_{8}$ etching process with a simultaneous $\mathrm{SF}_{6}$ passivation process, is performed to obtain smooth, vertical sidewalls. The resonator is released through wet etching of the underlying silicon dioxide layer, followed by critical point drying.

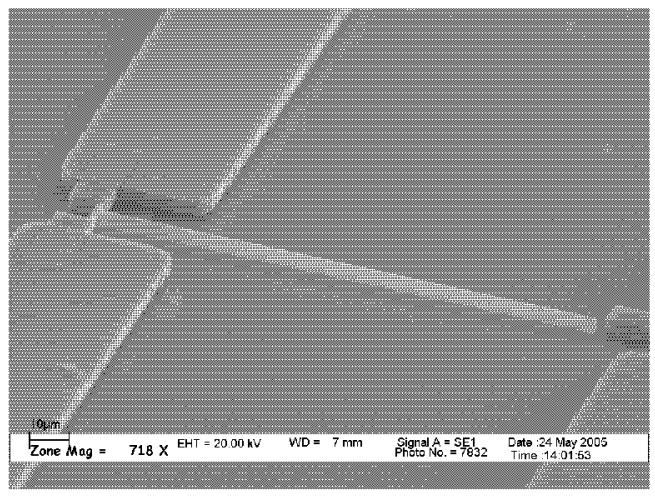

Fig. 1 SEM image of the lateral vibration T-shape microresonator (tilted). The free end of the resonator is approximately $2 \mu \mathrm{m}$ away from the driving electrode. The width and the height of the resonator are $1.1 \mu \mathrm{m}$ and $3 \mu \mathrm{m}$, respectively, and the lengths are $9 \mu \mathrm{m}$ for the base of the T-shape resonator and $127 \mu \mathrm{m}$ for the cantilever extending from the base.

The characterization of the resonator was done in a probe station with a variable pressure sample chamber. Fig. 2 shows the resonance curves of the resonator in a methane environment with pressures of $0.01,0.1,1,10,30,100,300$ 
and 760 Torr. As shown in the inset, the resonance frequency increases in a small amount as the pressure increases from 0.01 to 1 Torr, then falls in a relatively large amount from 1 to 10 Torr and continue decreases as the pressure increases from 1 Torr to atmospheric pressure.

Of primary interest is the regime above 10 Torr, the viscous damping regime, where the resonance frequency shift is due to the inertial force of the gas on the resonator. In Fig. 3, the shift in resonance frequency in the viscous damping regime is plotted as a function of pressure for four different gases: helium, methane, nitrogen and argon, with molar masses of 4, 16,28 and $40 \mathrm{~g} / \mathrm{mol}$, respectively. The results indicate a clear dependence of the resonance frequency shift on the molar mass of the gas. The frequency shift is observed to increase as the molar mass increases. For the resonator to function as a gas sensor, it must be possible to extract the molar mass from the resonance frequency shift. In the inset to Fig. 3, the relative resonance frequency shift is plotted as a function of the molar mass of the gas at a pressure of 760 torr. The resonant frequency shift changes from $0.25 \%$ (or $2.5 \%$ ) to $0.71 \%$ (or $7.1 \%$ ) as the molar mass increases from 4 to $40 \mathrm{~g} / \mathrm{mol}$, respectively.

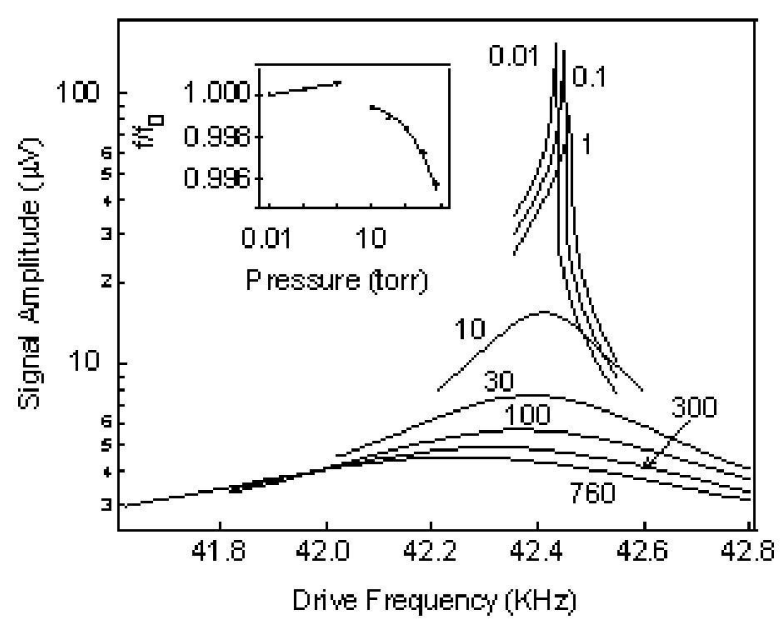

Fig. 2 Resonance frequency response curves of a T-shape resonator for pressures of $0.01,0.1,1,10,30,100,300,760$ Torr in a methane environment. Inset: relationship between relative resonance frequency and pressure for a methane environment.

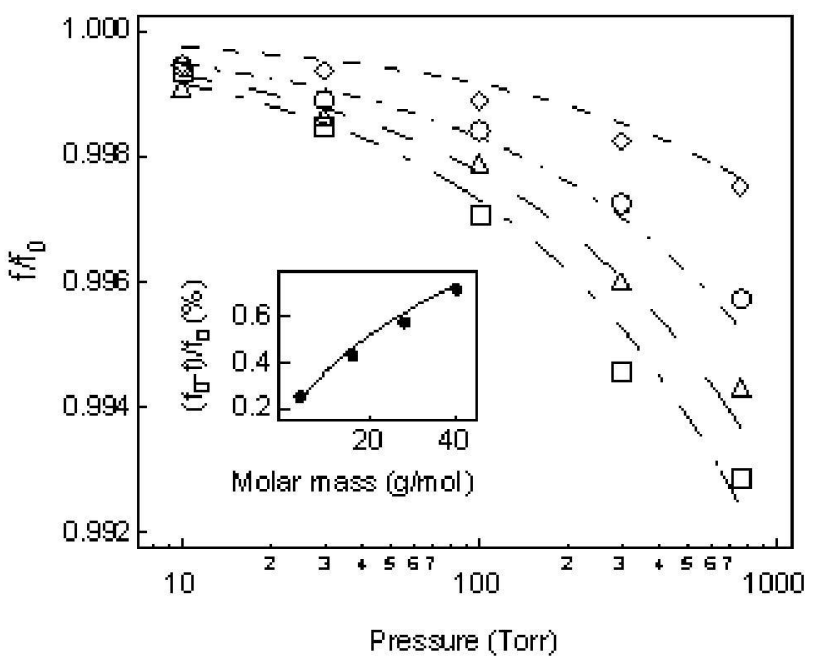

Fig. 3 The relative resonance frequency as a function of pressure from 10 to 760 Torr in helium(II), methane(ш), nitrogen( $\Delta$ ) and argon(II) environments. Inset: the relative resonance frequency shift of a resonator for the four gases at atmospheric pressure.

The increase in resonance frequency in the low pressure regime has not been reported in resonators using an optical detection method $[8,9]$, suggesting that it is related to our piezoresistive detection technique. The relation between the DC bias for piezoresistive detection and the resonance frequency is characterized (Fig. 4). The resonance frequency shift shows a clear linear dependence on the square of the DC bias (inset of Fig. 4). The temperature of the base beam of the T-resonator with different $\mathrm{DC}$ bias, 0 to $12 \mathrm{~V}$, is also measured using Raman spectra (Fig. 5). The temperature increase of $180^{\circ} \mathrm{K}$ is observed on the base beam of the resonator when DC bias is increased from $0 \mathrm{~V}$ to $10 \mathrm{~V}$ (Fig. 6). The temperature coefficient of Young's modulus, calculated from data of Fig. 4 and Fig. 5 , is $-93 \mathrm{ppm} /{ }^{\circ} \mathrm{C}$, which is close to the $-82 \mathrm{ppm} /{ }^{\circ} \mathrm{C}$, reported by $\mathrm{Li}$ et al [10]. This verifies that the resonance frequency shift with the DC bias is mainly due to the Young's modulus change from the heating effect. For the increase in resonance frequency in the low pressure regime (Fig. 2), as the pressure increases, the excess heat dissipates more easily through the gaseous environment, lowering the temperature of the cantilever relative to its temperature in vacuum, which results the increase in the resonance frequency that we observe. 


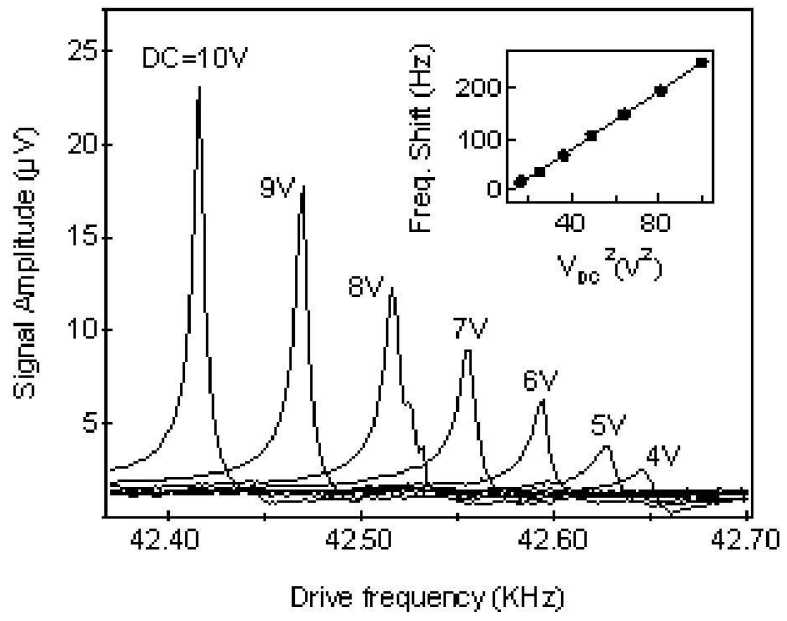

Fig. 4 The resonance response curves of a resonator with different DC bias applied on the base beam of the resonator. Inset: the resonance frequency shift of a resonator verses the square of the DC bias.

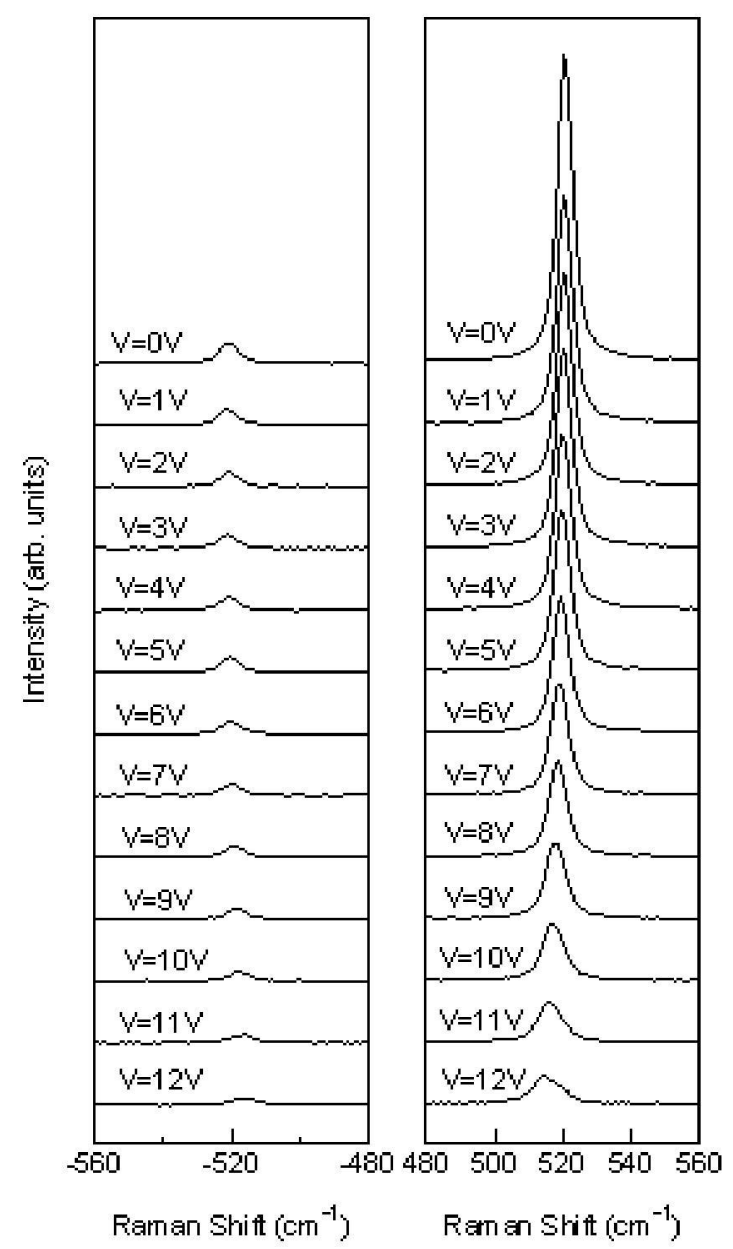

Fig. 5 The anti-stokes (left) and stokes (right) bands of Raman spectra of a resonator with different DC bias applied on the base beam.

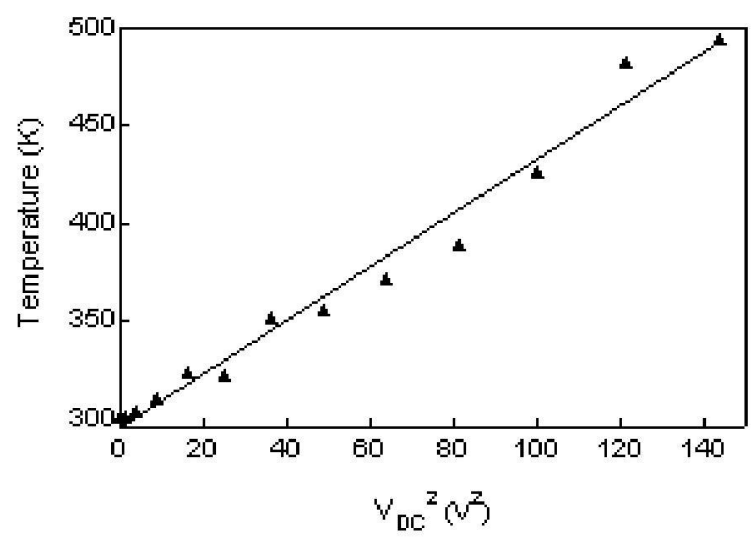

Fig. 6 The temperature, obtained from Raman spectra, of the base beam of the resonator verses the square of the DC bias applied on the base beam.

There is a relatively large drop in resonance frequency as the pressure goes from 1 to 10 torr, and a corresponding decrease in the amplitude of the vibration, as shown in Fig. 2. When the amplitude of the vibration is small, the restoring force varies linearly with the cantilever displacement. When the amplitude increases, an additional nonlinear term, which is proportional to the cube of the displacement, is added to the restoring force. The linear term is due to the bending moment of the cantilever, while the nonlinear term is due to the stretching of the resonator. This nonlinear restoring force results in bistability of the resonator and the shift of the peak of the response curve. The resonator was tested in vacuum with a series of different drive voltages to determine the influence of the nonlinear effect on our measurement. For drive bias larger than $8 \mathrm{~V}$, the bistability phenomenon, the rapidly switch from the largest amplitude to a lower amplitude, is observed (Fig. 7). The shift of the resonance frequency is found to increase with increasing driving voltage (or oscillation amplitude), as shown in inset of Fig. 7. This nonlinear effect can also account for the shift in resonance frequency observed with increasing pressure. 


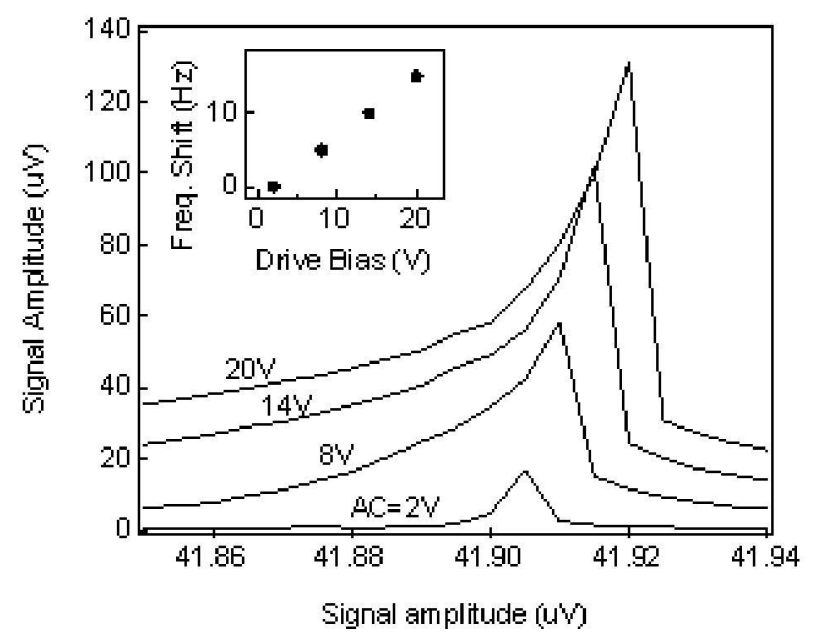

Fig. 7 The resonance response curves of a resonator with different Drive bias. Inset: the resonance frequency shift with the drive bias.

In summary, we have demonstrated the influence of gases with different molar mass on the resonance frequency of a lateral vibrating silicon resonator in the viscous damping regime. The influences of temperature and amplitude of the vibration on resonance frequency shift of the resonator are also given. The fabrication of the resonator is simple and the operation of the device is easy, reliable and repeatable. Because the detection method has a non-chemical basis, gas sensing is not limited by the availability of reaction mechanisms.

\section{ACKNOWLEDGMENT}

We thank Prof. Tim Dowling for providing the original motivation for this work and Mehdi Yazdanpanah for useful discussions. Funding provided by NSF / ONR (No. ECS0224114 ), NSF (No. EPS-0447479), NSF-EPSCOR (No. $6016955)$ and DOE-EPSCOR (No. 46411101095).

\section{REFERENCES}

[1] T. Thundat, E. A. Wachter, S. L. Sharp and R. J. Warmack, "Detection of mercury vapor using resonating microcantilevers", Appl. Phys. Lett. 661695 (1995)

[2] P. G. Datskos and I. Sauers, "Detection of 2-mercaptoethanol using gold-coated micromachined cantilevers", Sens. Actuators B, 6175 (1999)

[3] X. M. H. Huang, M. Manolidis, S. C. Jun, and J. Hone, "Nanomechanical hydrogen sensing", Appl. Phys. Lett. 86, 143104 (2005).

[4] M. Tortonese, R. C. Barrett, and C. F. Quate, "Atomic resolution with an atomic force microscope using piezoresistive detection", Appl. Phys. Lett. 62,834 (1993).

[5] J. A. Harley, and T. W. Kenny, "High-sensitivity piezoresistive cantilevers under 1000 A thick ", Appl. Phys. Lett. 75, 289 (1999).

[6] I. Bargatin, E. B. Myers, J. Arlett, B. Gudlewski, and M. L. Roukes, "Sensitive detection of nanomechanical motion using piezoresistive signal downmixing ", Appl. Phys. Lett. 86, 133109 (2005).

[7] M. Gel, and I. Shimoyama, "Force sensing submicrometer thick cantilevers with ultra-thin piezoresistors by rapid thermal diffusion", J. Micromech. Microeng. 14, 423 (2004).

[8] R. Sandberg, W Svendsen, K. Molhave and A. Boisen, "Temperature and pressure dependence of resonance in multi-layer microcantilevers", J. Micromech. Microeng. 15, 1454 (2005).

[9] S. J. O'Shea, P. Lu, F. Shen, P. Neuzil, and Q. X. Zhang, "Out-of-plane electrostatic actuation of microcantilevers", Nanotechnology 16, 602 (2005).

[10] X. Li, T. Ono, Y. Wang, and M. Esashi, "Ultrathin single-crystallinesilicon cantilever resonators: Fabrication technology and significant specimen size effect on Young's modulus", Appl. Phys. Lett. 83, 3081 (2003). 